\title{
Rainfall-Runoff Modeling Using NRCS-CN method and GIS approach
}

\author{
Rekha Verma ${ }^{1}$, Azhar Husain ${ }^{1}$, and Mohammed Sharif ${ }^{1}$ \\ ${ }^{1}$ Jamia Millia Islamia Central University
}

December 1, 2020

\begin{abstract}
Rainfall-Runoff modeling is a hydrological modeling which is extremely important for water resources planning, development, and management. In this paper, Natural Resource Conservation Service-Curve Number (NRCS-CN) method along with Geographical Information System (GIS) approach was used to evaluate the runoff resulting from the rainfall of four stations, namely, Bilodra, Kathlal, Navavas and Rellawada of Sabarmati River basin. The rainfall data were taken for 10 years (2005-2014). The curve number which is the function of land use, soil and antecedent moisture condition (AMC) was generated in GIS platform. The CN value generated for AMC- I, II and III were 57.29, 75.39 and 87.77 respectively. Using NRCS-CN method, runoff depth was calculated for all the four stations. The runoff depth calculated with respect to the rainfall for Bilodra, Kathlal, Navavas and Rellawada shows a good correlation of 0.96. The computed runoff was compared with the observed runoff which depicted a good correlation of $0.73,0.70,0.76$ and 0.65 for the four stations. This method results in speedy and precise estimation of runoff from a watershed.
\end{abstract}

\section{Hosted file}

Rainfall-Runoff Modeling Using NRCS-CN method and GIS approach.pdf available at https: //authorea.com/users/380325/articles/496365-rainfall-runoff-modeling-using-nrcs-cnmethod-and-gis-approach 


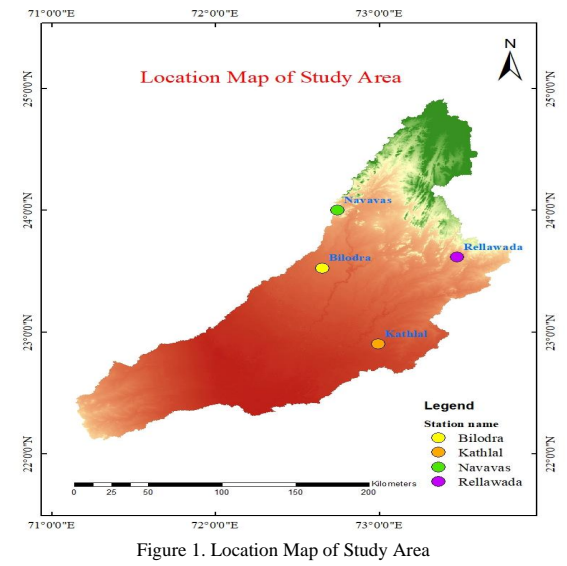




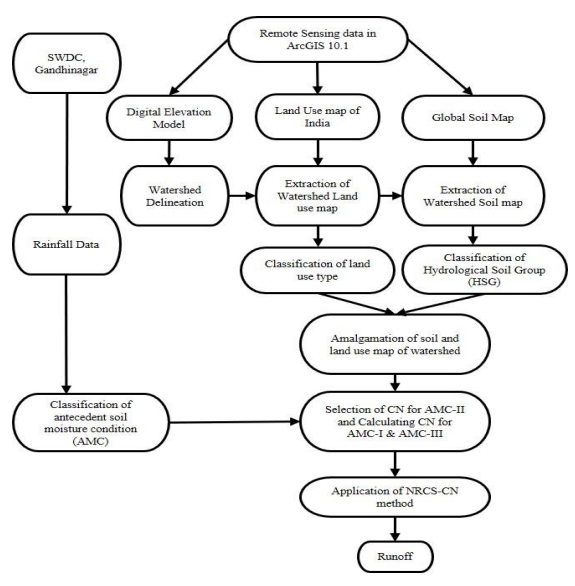

Figure 2 Flowchart of Proposed Model 


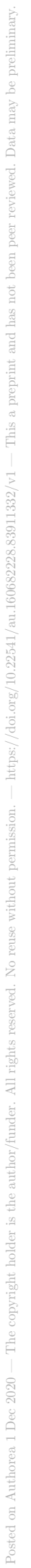

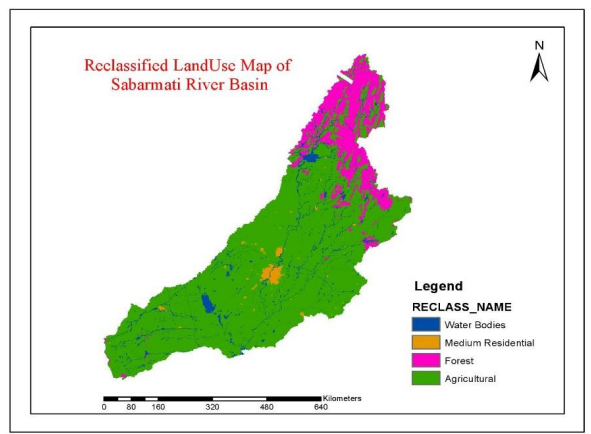

Figure 3: Reclassified LULC Map of Watershed

4 
HSG Soil Map of Sabarmati River Basin

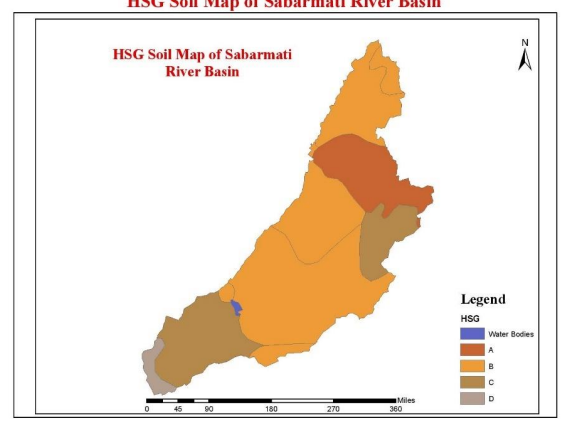

Figure 4: HSG Soil Map of Watershed 


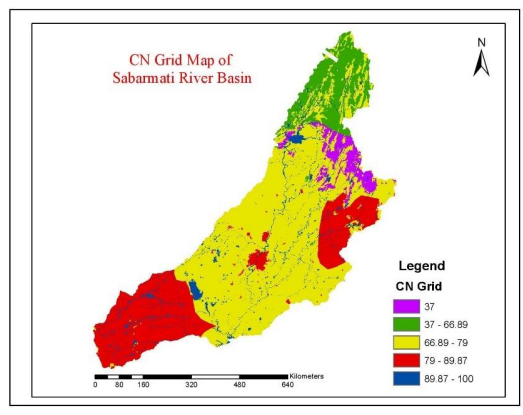

Figure 5: CN Grid Map of Watershed 


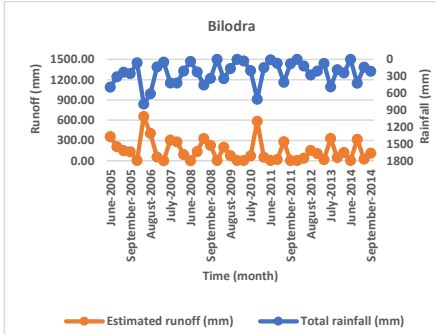

(a)

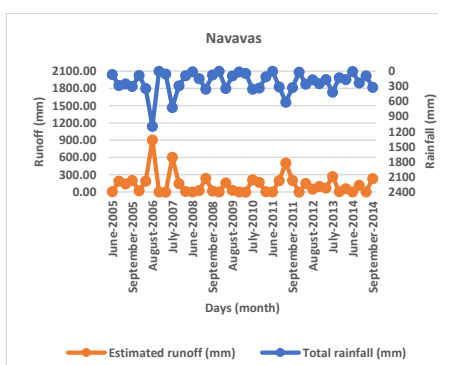

(c)

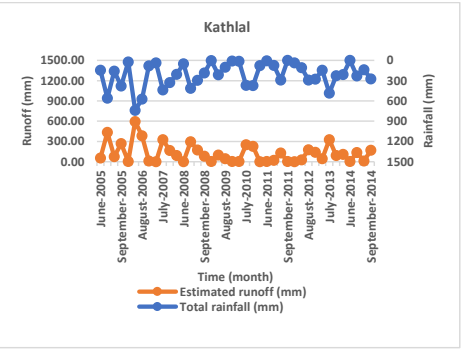

(b)

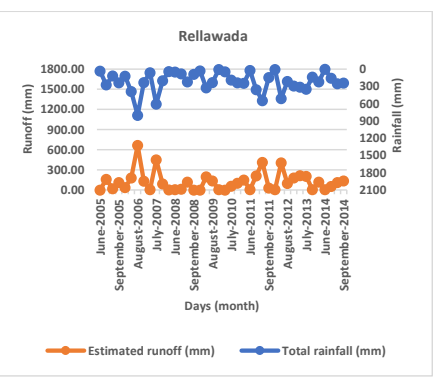

(d)

Figure 6: Rainfall and Estimated Runoff (a) Bilodra station (b) Kathlal station (c) Navavas station (d) Rellawada 

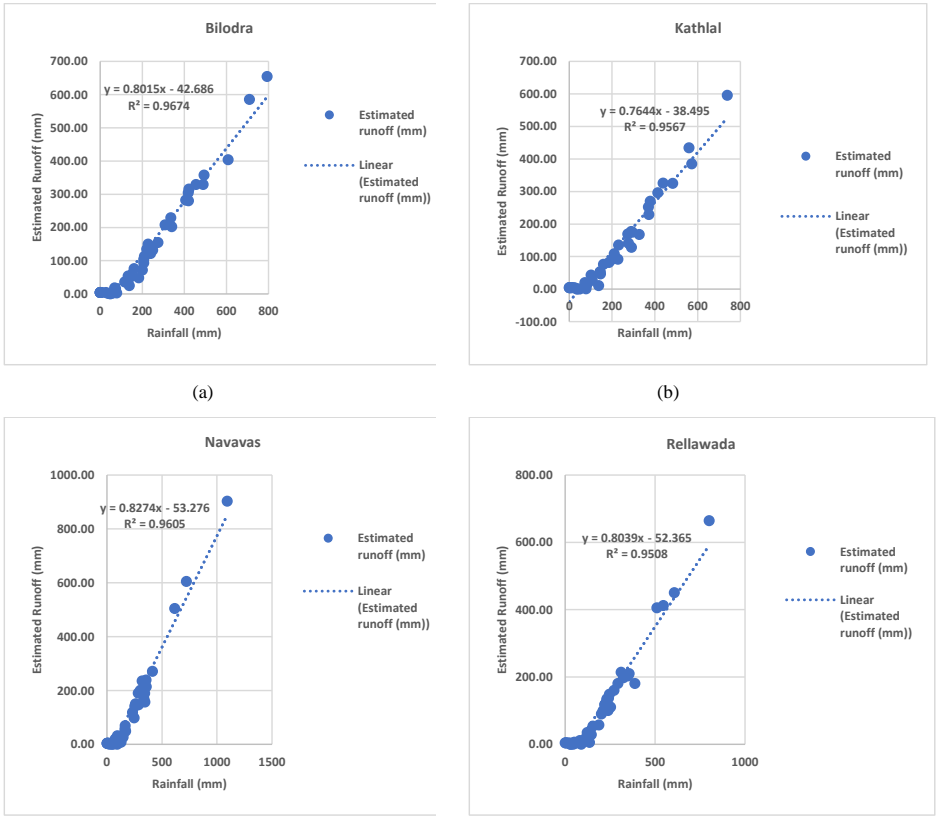

(c)

(d)

Figure 7: Scatter plot between Rainfall and Estimated Runoff (a) Bilodra station (b) Kathlal station (c) Navavas station (d) Rellawada station 


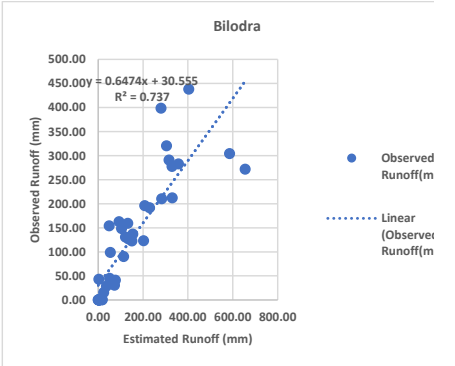

(a)

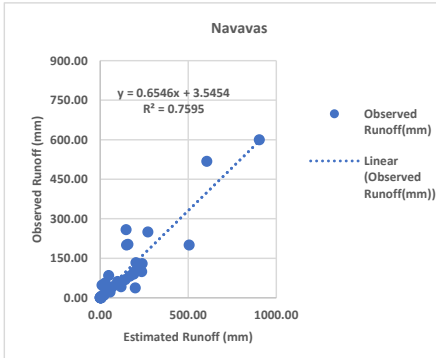

(c)

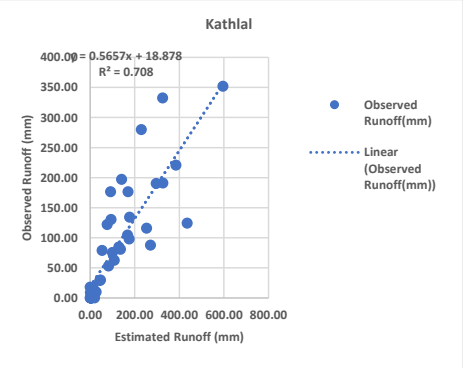

(b)

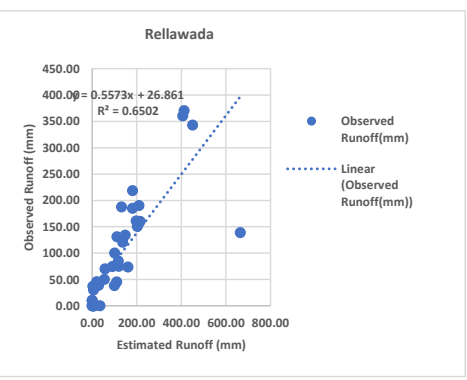

(d)

Figure 8: Scatter plot between Estimated and Observed Runoff (a) Bilodra station (b) Kathlal station (c) Navavas station (d) Rellawada station 
Table I Data required for modelling and their Sources

\begin{tabular}{|c|c|}
\hline Data & Source \\
\hline DEM & $\begin{array}{c}\text { SRTM 90m (CGIAR - CSI) } \\
\text { http://srtm.csi.cgiar.org/ }\end{array}$ \\
\hline Soil Map & FAO \\
& $\begin{array}{c}\text { http://www.fao.org/soils-portal/soil-survey/soil-maps-and- } \\
\text { databases/harmonized-world-soil-database-v12/en/ }\end{array}$ \\
\hline Land Use & Decadal Land Use and Land Cover Classification across India \\
https://daac.ornl.gov/VEGETATION/guides/Decadal_LULC_India.html & State Water Data Centre (SWDC), Gandhinagar \\
Indian Meteorological Department, Pune \\
India - Water Resources Information System \\
\hline Data
\end{tabular}


Table II SCS Hydrologic Soil Group (USDA)

\begin{tabular}{|c|l|l|l|l|c|}
\hline Group & Properties & Soil Textures & $\begin{array}{l}\text { Runoff } \\
\text { Potential }\end{array}$ & $\begin{array}{l}\text { Water } \\
\text { Transmission }\end{array}$ & $\begin{array}{l}\text { Minimum Rate } \\
\text { of Infiltration } \\
\text { (mm/hr) }\end{array}$ \\
\hline A & $\begin{array}{l}\text { Deep well drained } \\
\text { soils }\end{array}$ & $\begin{array}{l}\text { Sand, sandy loam, } \\
\text { loamy sand }\end{array}$ & Low & $\begin{array}{l}\text { High rate }(0.3 \\
\text { in/hr) }\end{array}$ & $>7.62$ \\
\hline B & $\begin{array}{l}\text { Moderately deep, } \\
\text { well drained with } \\
\text { moderately fine to } \\
\text { coarse texture }\end{array}$ & Loam or silt loam & Moderate & $\begin{array}{l}\text { Moderate rate } \\
(0.15-0.3 \mathrm{in} / \mathrm{hr})\end{array}$ & $3.81-7.62$ \\
\hline C & $\begin{array}{l}\text { Soils with moderately } \\
\text { fine to fine texture }\end{array}$ & Sandy clay loam & Moderate & $\begin{array}{l}\text { Low rate }(0.05- \\
0.15 \text { in/hr) }\end{array}$ & $1.27-3.81$ \\
\hline D & $\begin{array}{l}\text { Soil which swell } \\
\text { significantly when } \\
\text { wet, heavy plastic } \\
\text { and soil with a } \\
\text { permanent high-water } \\
\text { table }\end{array}$ & $\begin{array}{l}\text { Clay, clay loam, } \\
\text { sandy clay, silty } \\
\text { clay, silty clay } \\
\text { loam }\end{array}$ & High & $\begin{array}{l}\text { Very low rate } \\
(0-0.05 i n / h r)\end{array}$ & $0-1.27$ \\
\hline
\end{tabular}


Table III Classification of Antecedent Soil Moisture Condition

\begin{tabular}{|c|l|c|c|}
\hline \multirow{2}{*}{ AMC } & \multicolumn{2}{|c|}{ Properties of Soils } & \multicolumn{2}{c|}{$\begin{array}{c}\text { Total 5-day antecedent } \\
\text { rainfall }(\mathrm{mm})\end{array}$} \\
\cline { 3 - 4 } & & Dormant Season & $\begin{array}{c}\text { Growing } \\
\text { season }\end{array}$ \\
\hline I & $\begin{array}{l}\text { Soils are dry (soil moisture content is at wilting } \\
\text { point) }\end{array}$ & $<13 \mathrm{~mm}$ & $<36 \mathrm{~mm}$ \\
\hline II & Average Condition & $13-28 \mathrm{~mm}$ & $36-53 \mathrm{~mm}$ \\
\hline III & $\begin{array}{l}\text { Soils are saturated from antecedent rainfalls (soil } \\
\text { moisture content is at field capacity) }\end{array}$ & $>28 \mathrm{~mm}$ & $>53 \mathrm{~mm}$ \\
\hline
\end{tabular}


Table- IV Curve Number of Sabarmati river basin under AMC-II, AMC-I \& AMC-III

\begin{tabular}{|c|c|c|c|c|c|c|c|}
\hline \multicolumn{8}{|c|}{ Curve Number } \\
\hline Land Use & HSG & Area $(\mathrm{km} 2)$ & $\mathrm{CN}$ & CN*Area & CNw(II) & $\mathrm{CN}(1)$ & CN(III) \\
\hline Water Bodies & - & 1371.13487 & 100 & 137113.49 & \multirow[t]{13}{*}{75.39} & \multirow[t]{13}{*}{\begin{tabular}{|l|}
57.29 \\
\end{tabular}} & \multirow[t]{13}{*}{87.77} \\
\hline \multirow{4}{*}{$\begin{array}{c}\text { Medium } \\
\text { Residential }\end{array}$} & A & 7.893683 & 76 & 599.91991 & & & \\
\hline & B & 355.694355 & 85 & 30234.02 & & & \\
\hline & $\mathrm{C}$ & 26.930029 & 90 & 2423.7026 & & & \\
\hline & $\mathrm{D}$ & 1.274995 & 92 & 117.29954 & & & \\
\hline \multirow[t]{4}{*}{ Forest } & A & 1283.07424 & 37 & 47473.747 & & & \\
\hline & B & 2884.83287 & 60 & 173089.97 & & & \\
\hline & $\mathrm{C}$ & 128.333441 & 73 & 9368.3412 & & & \\
\hline & D & 22.7169 & 79 & 1794.6351 & & & \\
\hline \multirow[t]{4}{*}{ Agricultural } & $\mathrm{A}$ & 2654.15283 & 67 & 177828.24 & & & \\
\hline & B & 14665.1308 & 77 & 1129215.1 & & & \\
\hline & $\mathrm{C}$ & 6327.6183 & 83 & 525192.32 & & & \\
\hline & D & 595.745791 & 87 & 51829.884 & & & \\
\hline
\end{tabular}


Table- V Estimated annual runoff depth for annual rainfall using NRCS-CN

\begin{tabular}{|c|c|c|c|c|c|c|c|c|}
\hline \multirow{2}{*}{ Year } & \multicolumn{2}{|c|}{ Rellawada } & \multicolumn{2}{c|}{ Bilodra } & \multicolumn{2}{c|}{ Kathlal } & \multicolumn{2}{c|}{ Navavas } \\
\cline { 2 - 10 } & $\begin{array}{c}\text { Rainfall } \\
(\mathrm{mm})\end{array}$ & $\begin{array}{c}\text { Runoff } \\
(\mathrm{mm})\end{array}$ & $\begin{array}{c}\text { Rainfall } \\
(\mathrm{mm})\end{array}$ & $\begin{array}{c}\text { Runoff } \\
(\mathrm{mm})\end{array}$ & $\begin{array}{c}\text { Rainfall } \\
(\mathrm{mm})\end{array}$ & $\begin{array}{c}\text { Runoff } \\
(\mathrm{mm})\end{array}$ & $\begin{array}{c}\text { Rainfall } \\
(\mathrm{mm})\end{array}$ & $\begin{array}{c}\text { Runoff } \\
(\mathrm{mm})\end{array}$ \\
\hline 2005 & 657.5 & 291.97 & 1280 & 847.16 & 1243.5 & 835.21 & 905 & 538.15 \\
\hline 2006 & 1544.5 & 1011.95 & 1593 & 1114.54 & 1417 & 996.73 & 1518 & 1118 \\
\hline 2007 & 912 & 549 & 1093.5 & 678.56 & 1009.5 & 587.46 & 1152 & 763 \\
\hline 2008 & 442 & 137 & 1050 & 694.85 & 944 & 553.68 & 586 & 289.1 \\
\hline 2009 & 590 & 338 & 501.5 & 286.87 & 326.5 & 151.23 & 457.5 & 197.32 \\
\hline 2010 & 716.5 & 307.13 & 1081.5 & 712.62 & 832.5 & 487.19 & 848 & 390.35 \\
\hline 2011 & 1068 & 655.91 & 561.5 & 308.51 & 368.5 & 156.55 & 1253.5 & 911.18 \\
\hline 2012 & 1018 & 690.17 & 598 & 299.49 & 710.5 & 346.26 & 697 & 297.78 \\
\hline 2013 & 1016 & 541 & 985 & 516.67 & 1068 & 571.49 & 872.5 & 405.27 \\
\hline 2014 & 648 & 306.63 & 770 & 458.43 & 642 & 319.57 & 654.5 & 359 \\
\hline
\end{tabular}

